\title{
The Effect of Coronavirus Stress on Job Burnout in Nurses with the Moderating Role of Psychological Capital
}

\author{
Mohsen Arefnejad $^{1}$ (D), Fariborz Fathi Chegeni2* ${ }^{*}$, Mostafa Omidnejad ${ }^{3}$
}

1. Assistant Professor, Department of Management, Faculty of Management and Economics, Lorestan University, Khorramabad, Iran

2. PhD Student, Department of Management, Faculty of Management and Economics, Lorestan University, Khorramabad, Iran

3. Master Student, Department of Management, Faculty of Economics and Administrative Sciences, Malayer University, Hamadan, Iran

\begin{tabular}{ll}
\hline \multicolumn{1}{c}{ Article Info } & ABSTRACT \\
\cline { 1 - 1 } $\begin{array}{l}\text { Received: } 2021 / 04 / 11 ; \\
\text { Accepted: } 2021 / 08 / 22 ;\end{array}$ & $\begin{array}{l}\text { Background and Objectives: The nursing profession is basically stressful in } \\
\text { nature. In the meantime, the spread of the coronavirus and its rapid spread throughout } \\
\text { ePublished: } 2021 / 09 / 21\end{array}$ \\
$\begin{array}{l}\text { the country has caused the over-normal influx of patients infected with this virus to } \\
\text { hospitals and increased nurses' work stress. Therefore, the aim of this study was to } \\
\text { investigate the effect of Coronavirus Stress on Job burnout in nurses with the } \\
\text { and read the article online }\end{array}$ & $\begin{array}{l}\text { moderating role of psychological capital in Khorramabad Shohadaye Ashayer } \\
\text { Hospital, Iran. }\end{array}$
\end{tabular}

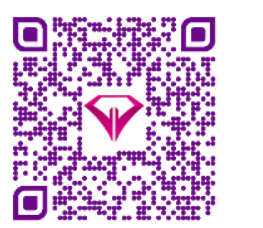

Corresponding Author Fariborz Fathi Chegeni PhD Student, Department of Management, Faculty of Management and Economics, Lorestan University, Khorramabad, Iran Email: arifth@gmail.com
Methods: The present study is descriptive research in field studies in terms of applied purpose; data collection was performed using surveys. The statistical population of the study was 230 nurses working in Khorramabad Shohadaye Ashayer Hospital, Iran. Based on Krejcie and Morgan table, a sample size of 140 people were selected by stratified random sampling method. To measure the variables of the research, a questionnaire was used which included three sections of Coronavirus Stress, Job burnout and psychological capital. The reliability of the questionnaire was confirmed using Cronbach's alpha method and its validity was confirmed using convergent validity. For data analysis, structural equation modeling and SPSS and PLS software were used.

Results: The results showed that at the $95 \%$ confidence level, Coronavirus Stress has a positive and significant effect on nurses' Job burnout and psychological capital has a moderating role in relation to Coronavirus Stress and Job burnout.

Conclusion: According to the findings, it can be said that Coronavirus Stress has a significant role in increasing nurses' Job burnout, and in the meantime, nurses with stronger psychological capital suffer less burnout than nurses with weaker psychological capital. Therefore, it is necessary to reduce the stress of Coronavirus disease by strengthening the psychological capital of nurses, which is a teachable component, and also equipping nurses with the best equipment.

Keywords: COVID 19, Nurses, Job burnout, Psychological capital

\section{How to Cite This Article:}

Arefnejad M, Fathi Chegeni F, Omidnejad M. The Effect of Coronavirus Stress on Job Burnout in Nurses with the Moderating Role of Psychological Capital. Iran J Ergon. 2021; 9(2):58-68 


\section{Extended Abstract}

\section{Introduction}

The nursing profession is basically stressful in nature. In the meantime, the spread of the coronavirus and its rapid spread throughout the country has caused the over-normal influx of patients infected with this virus to hospitals and increased nurses' work stress. Therefore, the aim of this study was to investigate the effect of Coronavirus Stress on Job burnout in nurses with the moderating role of psychological capital in Khorramabad Shohadaye Ashayer Hospital, Iran.

\section{Methods}

The present study is descriptive research in field studies in terms of applied purpose; data collection was performed using surveys. The statistical population of the study was 230 nurses working in Khorramabad Shohadaye Ashayer
Hospital, Iran. Based on Krejcie and Morgan table, a sample size of 140 people were selected by stratified random sampling method. To measure the variables of the research, a questionnaire was used, which included three sections of Coronavirus Stress, Job burnout, and psychological capital. The reliability of the questionnaire was confirmed using Cronbach's alpha method, and its validity was confirmed using convergent validity. For data analysis, structural equation modeling and SPSS and PLS software were used.

\section{Results}

The results showed that at the $95 \%$ confidence level, Coronavirus Stress has a positive and significant effect on nurses' Job burnout, and psychological capital has a moderating role in relation to Coronavirus Stress and Job burnout.

Table 1. Fitting of measurement models

\begin{tabular}{|cccc|}
\hline Variable & Cronbach's alpha & Combined reliability & $\begin{array}{c}\text { Average Variance } \\
\text { Extracted (AVE) }\end{array}$ \\
\hline Corona stress & 0.889 & 0.861 & 0.667 \\
\hline Job burnout & 0.857 & 0.817 & 0.740 \\
\hline Psychological capital & 0.796 & 0.768 & 0.639 \\
\hline
\end{tabular}

\section{Discussion}

The present study investigated the relationship between Corona pandemic stress and burnout in nurses of Khorramabad Nomadic Martyrs Hospital with a moderating role of psychological capital. For this purpose, two hypotheses were proposed. First, does the stress of Corona affect the burnout of nurses working in Khorramabad Nomad Martyrs Hospital? And second, whether nurses' psychological capital has a moderating role in correlating stress with COVID-19 and burnout?

Analyzing the first hypothesis, it can be said that although job stress is present in all jobs, but in professions that deal with human health, this issue is doubly essential [34]. In fact, nurses are constantly exposed to stress due to the nature of their profession [35]. Studies show that prolonged and persistent workplace stress leads to issues such as resignations, frequent absences, reduced energy and decreased work efficiency [36], reduced creativity and incompatibility with colleagues [37], decreased professional satisfaction [38], reduced quality of patient care [39], decreased correct and timely decisions, decreased ability to work and commitment of employees, feeling of inadequacy, depression, decreased job values, disgust and fatigue [40] and finally the occurrence of burnout syndrome [16].

Regarding the second hypothesis, it can be said that the rate of burnout due to the Corona pandemic in people with high psychological capital was lower than nurses with low psychological capital. Analyzing this hypothesis, it can be said that people with high psychological capital had higher morale and more hope, and their resilience while facing difficult and critical situations was higher than those with lower psychological capital. In fact, they are 
more likely to believe in their ability to cope with sudden problems and crises, and with a positive attitude toward the future and problems, they reduce the effect of stress on burnout. People with higher psychological capital hope that the crisis will end sooner or later and that they should not lose their temper. This positive view of problems strengthens their minds in dealing with problems and increases their tolerance for ambiguity and complex situateions. In this regard, the results of various studies show the effect of psychological capital on reducing pessimism, feelings of inadequacy [44], negative attitudes toward work [45] and absenteeism and leaving the workplace and maybe the rate of burnout under the influence of factors such as workplace pressures. Reduce feelings of helplessness, passimism, hopelessness, and feelings of inadequacy [46].

Hashemi Nosrat et al. showed that using psychological capital can eliminate psychological resistance and strengthen the defense against stress. Since stress is the leading cause of burnout, burnout can be reduced by increasing psychological capital [47]. Silva (2010) also confirms the role of psychological capital in increasing motivation and believes that increasing psychological capital increases hope and self-confidence and increases the amount of effort of individuals [48]. Nezamii and Giourian (2016) also showed in their research that psychological capital can be an excellent executive solution to reduce burnout [46].

Finally, according to the study results, hospital managers are recommended to provide the best prevention equipment and help nurses to reduce their stress of the Corona pandemic to some extent. It is suggested that by providing special facilities such as life insurance and work adaptation and nurses' salaries with the particular conditions that exist, to increase their satisfaction and hope. It is suggested that by providing the necessary infrastructure in the hospital to facilitate nurses' communication with their families virtually, they can strengthen their morale and reduce stress. Given that psychological capital is not a fixed variable and can be improved with practice, it is recommended that special counselors be used for strengthening psychological capital in nurses. Given that the stress of COVID-19 is both a weakening of the immune system and a negative psychological effect, hospital managers are advised to provide nurses with up-todate information on the functioning of the virus and to create empathy by increasing communication.

\section{Conclusion}

According to the findings, it can be said that Coronavirus Stress has a significant role in increasing nurses' Job burnout. In the meantime, nurses with more robust psychological capital suffer less burnout than nurses with weaker psychological capital. Therefore, it is necessary to reduce the stress of Coronavirus disease by strengthening the psychological capital of nurses, which is a teachable component and equipping nurses with the best equipment.

\section{Acknowledgement}

We cherish the memory of all the dear nurses who joined the health martyrs due to COVID-19 and all the managers and nurses of the different wards of the Khorramabad Nomad Martyrs Hospital who cooperated in conducting the research are highly appreciated and thanked.

\section{Conflict of Interest}

The authors declared no conflict of interest. 
اثر استرس ابتلا به كرونا بر فرسودَى شغلى يرستاران با نقش تعديلَّر سرمائُ روانشناختى بـى

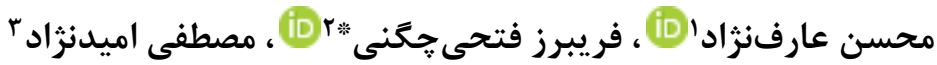

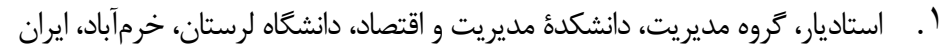

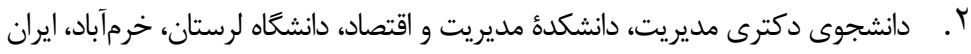

$$
\begin{aligned}
& \text { r". دانشجوى كارشناسىارشد مديريت، دانشكدة علوم اقتصادى و ادارى، دانشكاه ملاير، همدان، ايران }
\end{aligned}
$$

\begin{tabular}{|c|}
\hline خلاصه \\
\hline 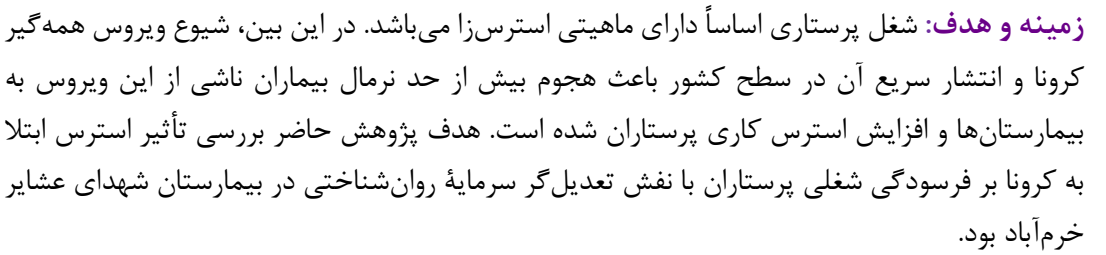 \\
\hline 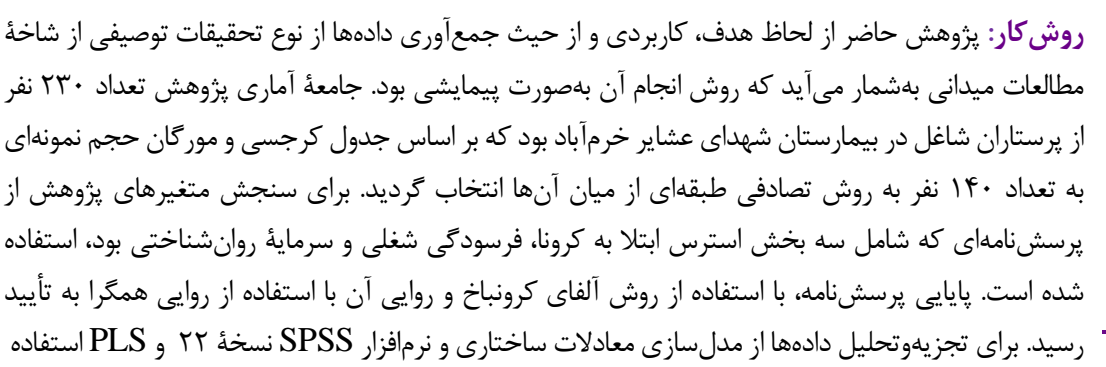 \\
\hline
\end{tabular}
شده است. يافتهها: نتايج نشاندهندة اين است كه در سطح اطمينان ه9 درصد استرس ابتلا به كرونا اثر مثبت و

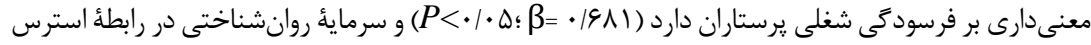

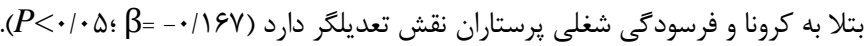
نتيجه كيرى: با توجه به يافتهها مىتوان كَفت كه استرس ابتلا به كرونا نقش بارزى در افزايش فرسودىى

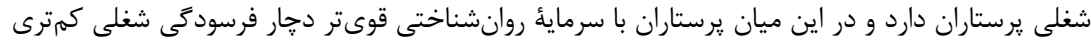

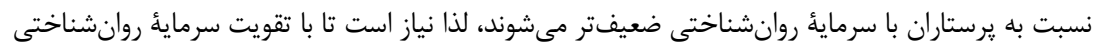

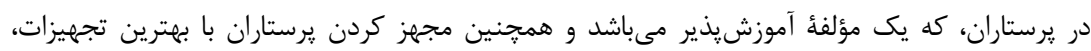
استرس ابتلا به كرونا را در آنها كاهش داد ماد.

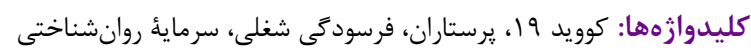

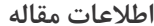

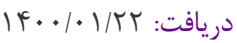

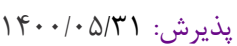

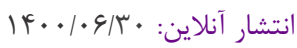

$$
\text { فويسندهُ مسئول: }
$$

دانشجوى دكترى مديريت، دانشكدو

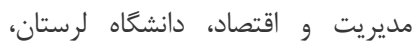

خرمآباد، ايران

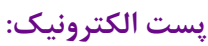

farifth@gmail.com

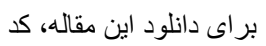

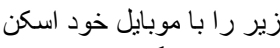
كنيد.

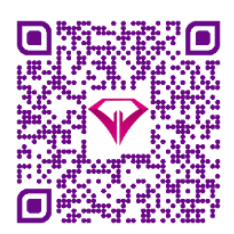

كبىرايت C) مجله اركونومى؛ دسترسى آزاد؛

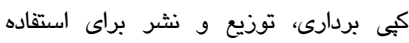
غيرتجارى با ذكر منبع آزاد است.

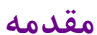

مجارى تنفسى و هم از طريق ذرات هوا از شخصى به شخص

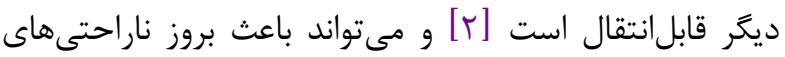

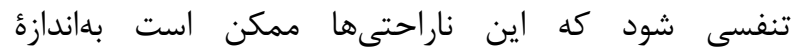

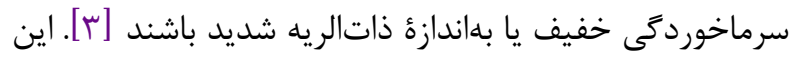
ويروس تقريباً در تمام كشورهاى جهان، جنبئ فراگير و
بحران جديدى ناشى از گسترش كروناويروس در اواخر

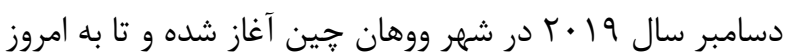

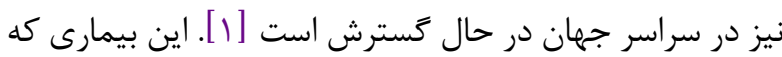

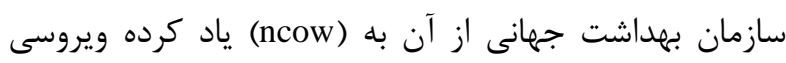

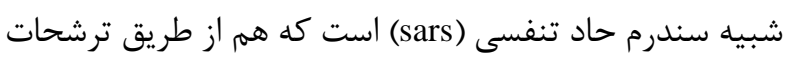




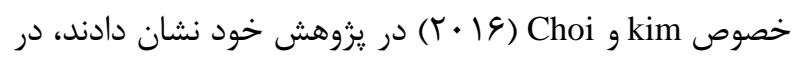

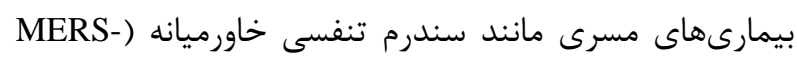

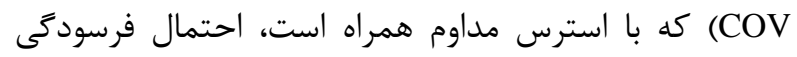

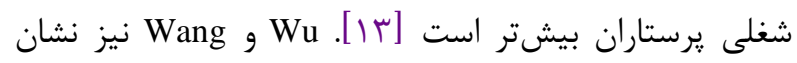

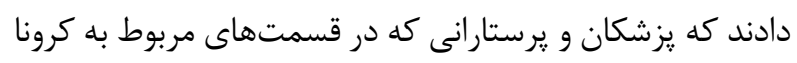

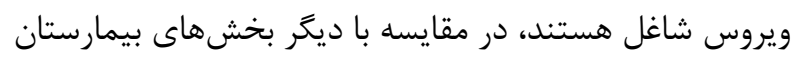

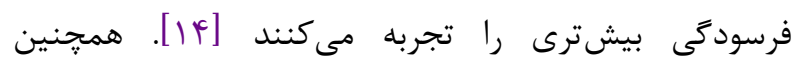

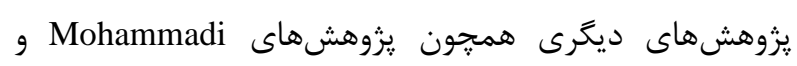

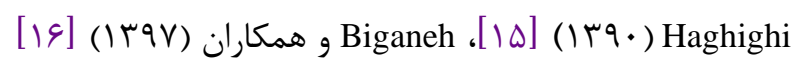

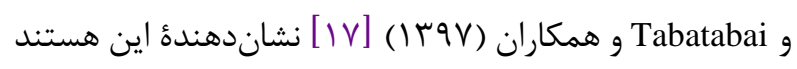

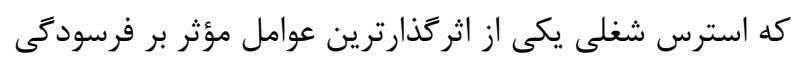

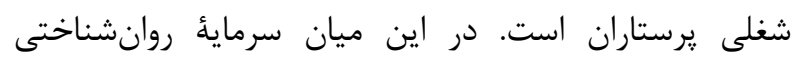

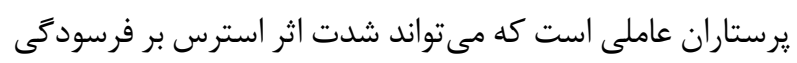

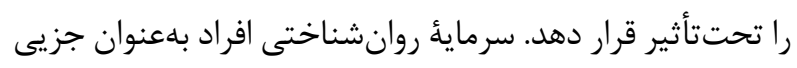

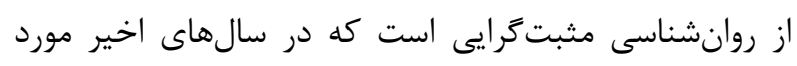

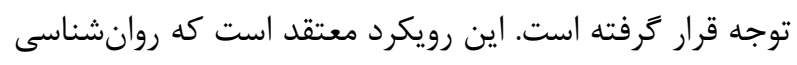

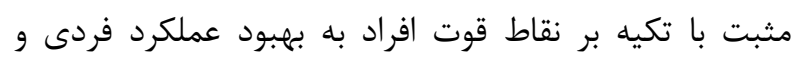

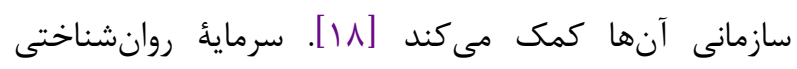

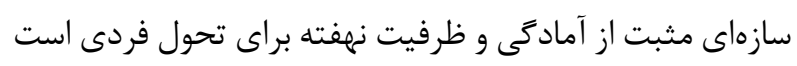

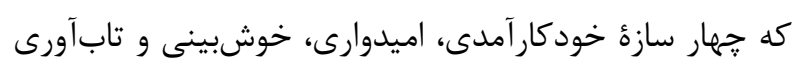

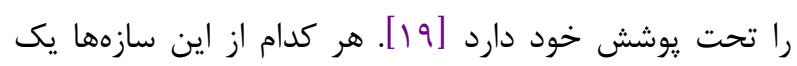

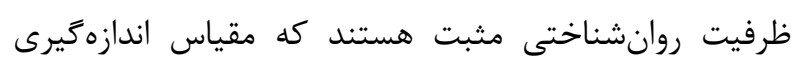

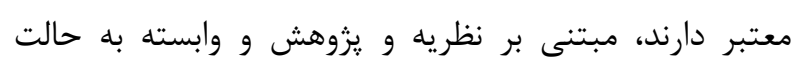

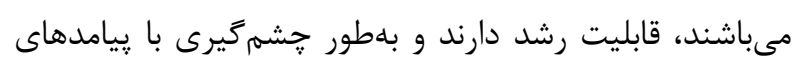

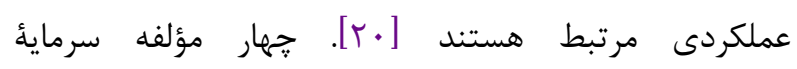

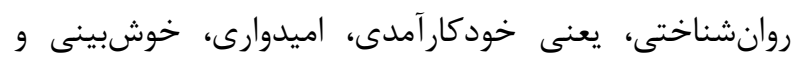

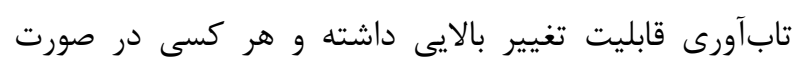

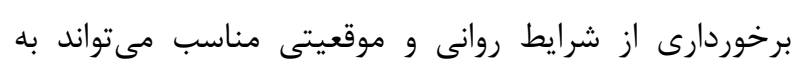

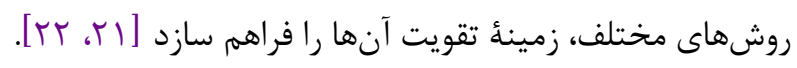

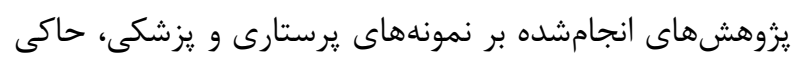

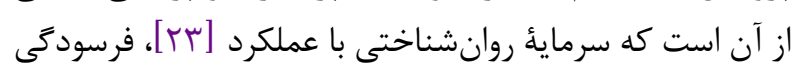

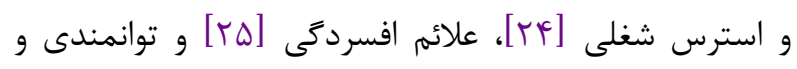

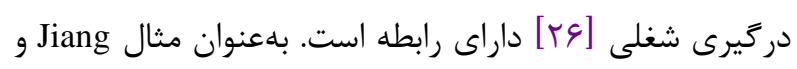

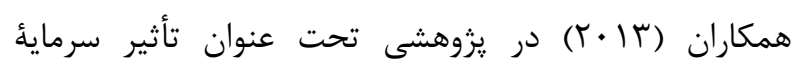
روانشناختى بر فرسودىى شغلى برستاران با نقش ميانجى تعهد

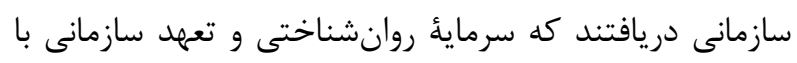

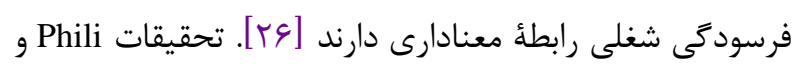

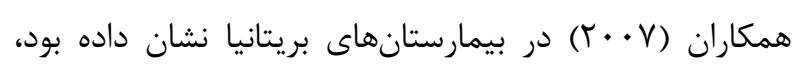

عالم گيرى به خود كرفته و تمام ملتهاى جهان را تحت تأثير

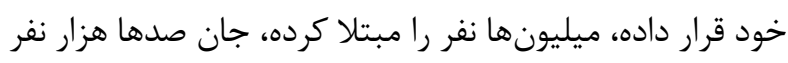

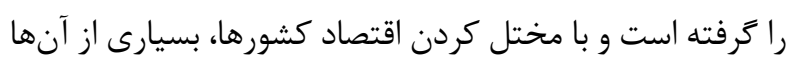

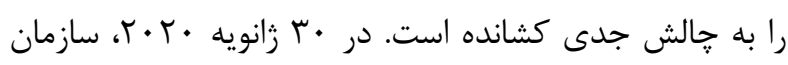

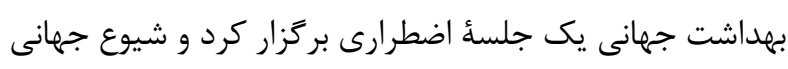

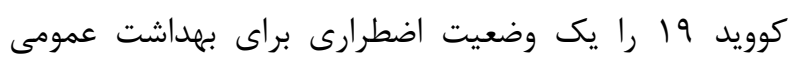

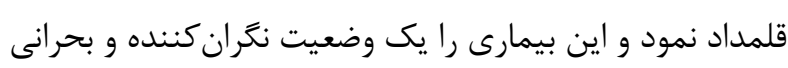

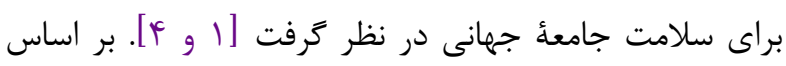

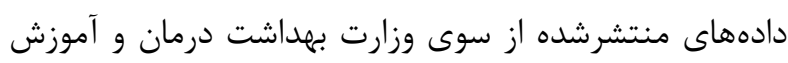

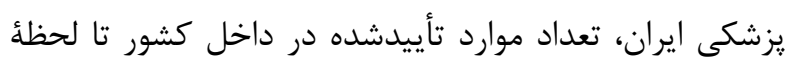

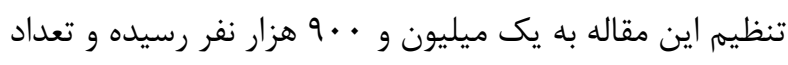

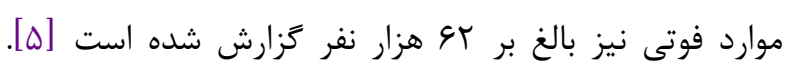

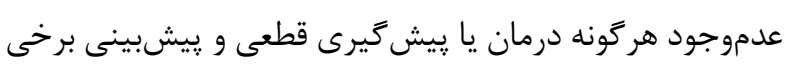

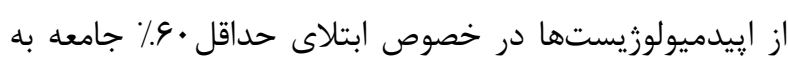

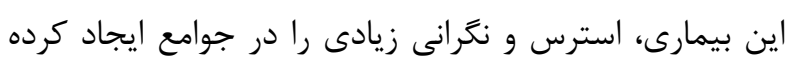

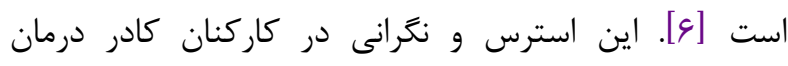

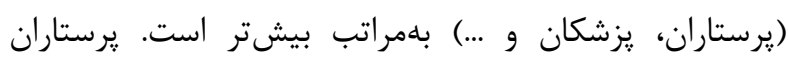

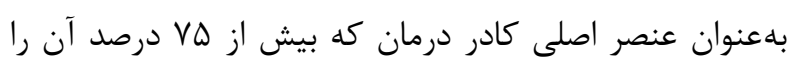

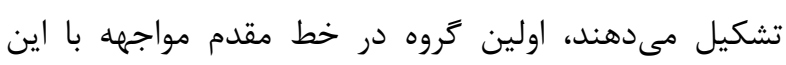

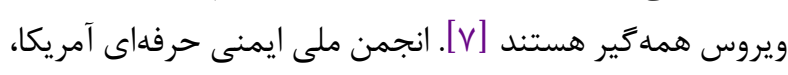

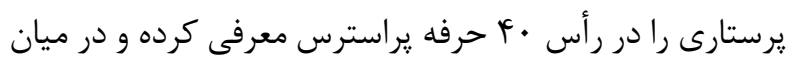

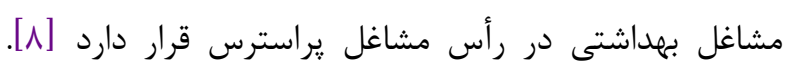

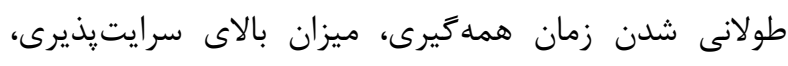

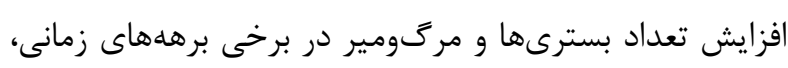

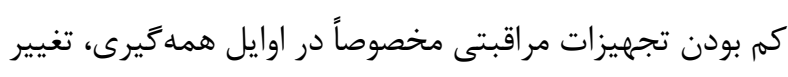

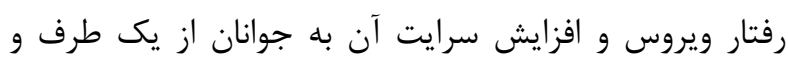

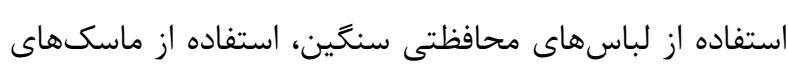

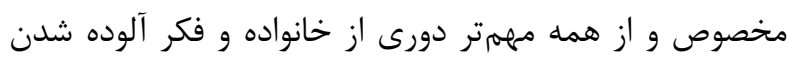

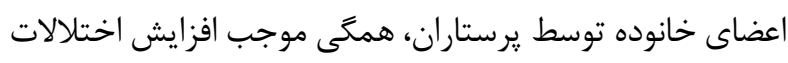

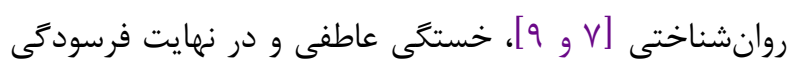

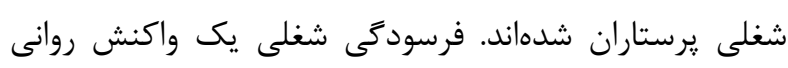

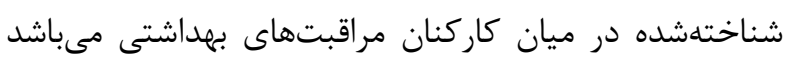

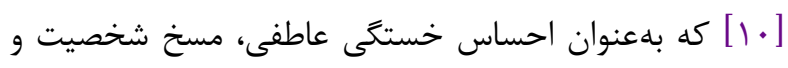

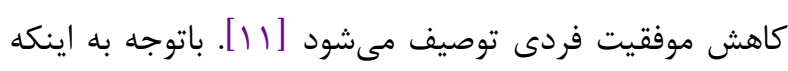

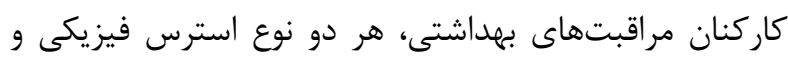

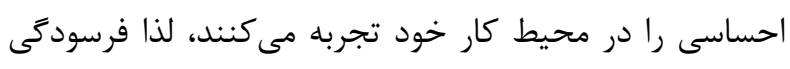

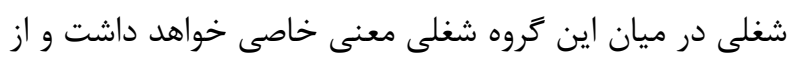

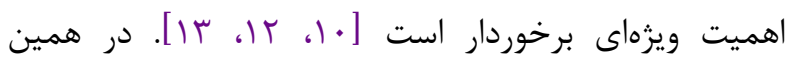




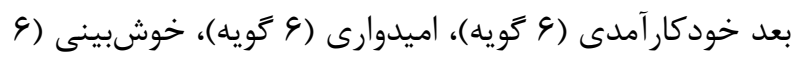

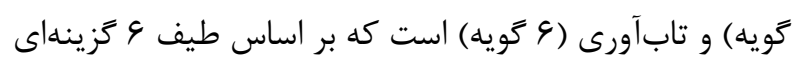

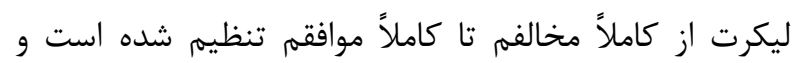

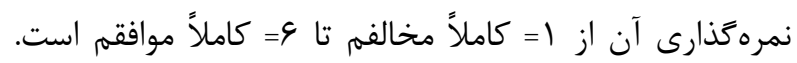

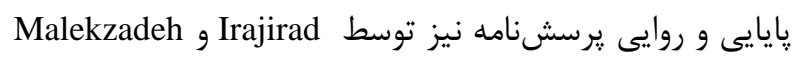

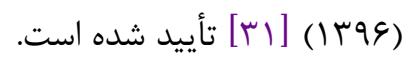

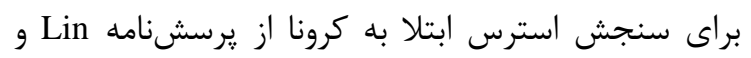

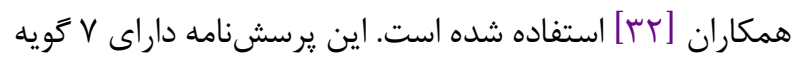

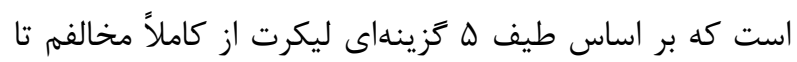

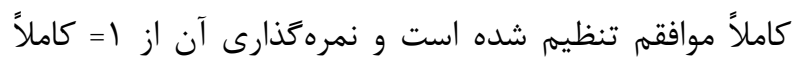

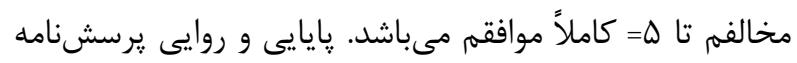

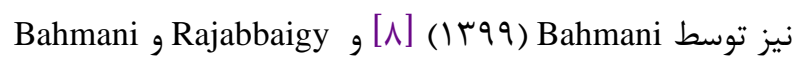

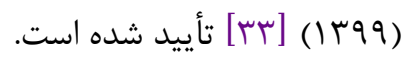

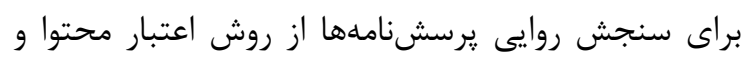

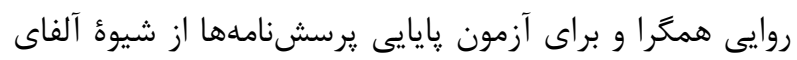

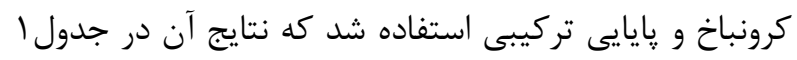

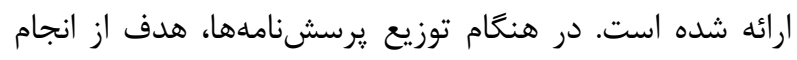

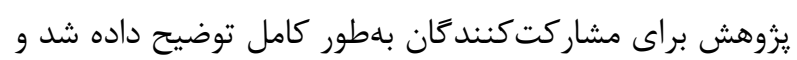

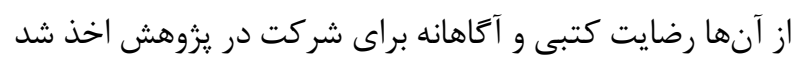

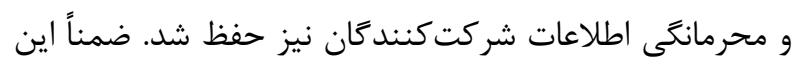

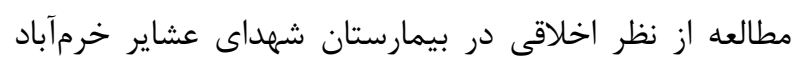

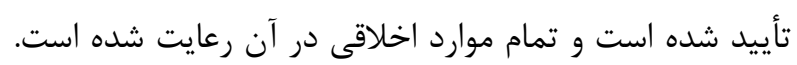

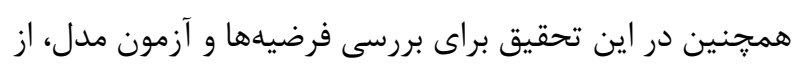
تكنيك مدلسازى معادلات ساختارى و نرمافزارهاى SPSS

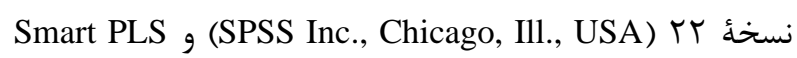
استفاده كرديده است.

\section{يافته ها}

در اين يزوهش براى بررسى برازش مدل مفهومى يزوهش و

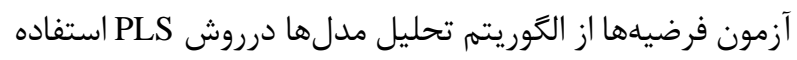

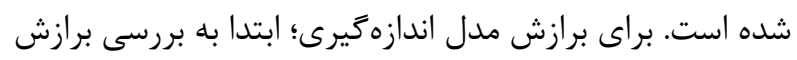

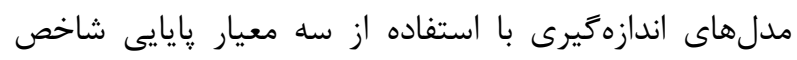

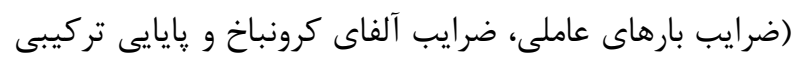

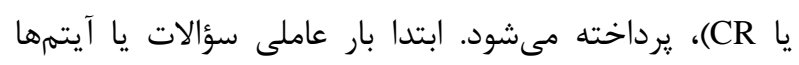

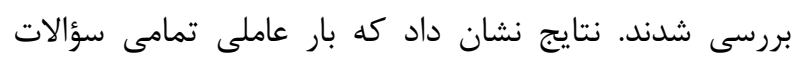

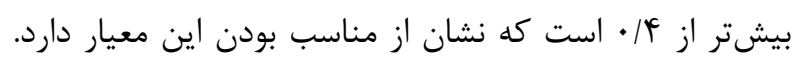

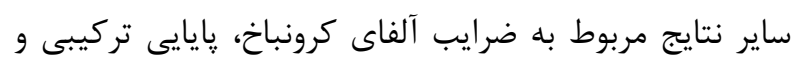
روايى همخرا در جدول شماره ال ارائه شده است.
يرستارانى كه در رويارويى با مشكلات حرفهاى جهت حفظ

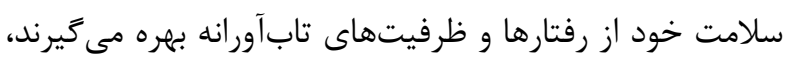

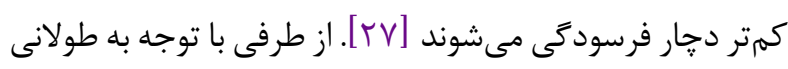

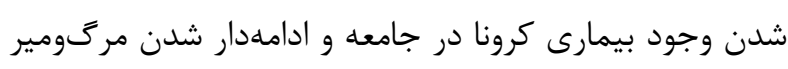

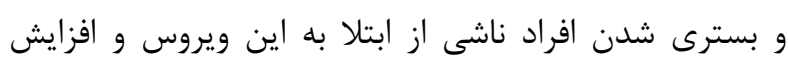

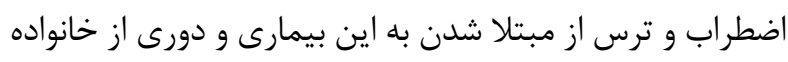

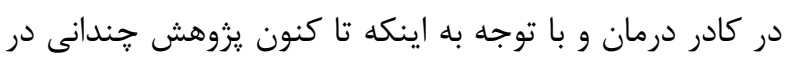

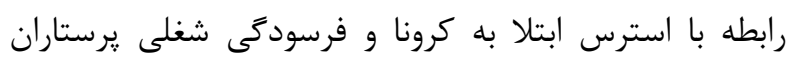

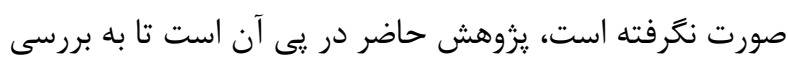

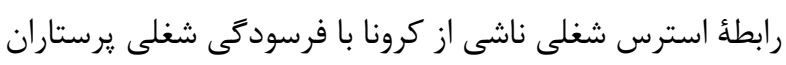

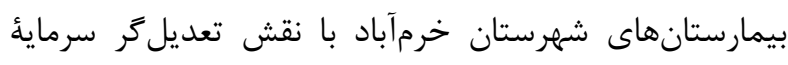
روانشناختى بيردازد.

روش شناسى

يزوهش حاضر از لحاظ هدف، كاربردى و از حيث جمعآورى

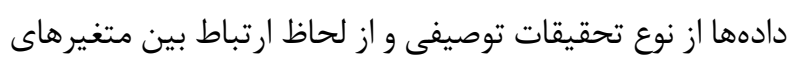

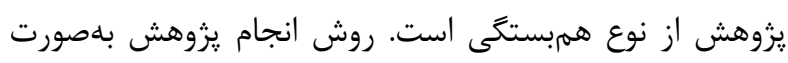

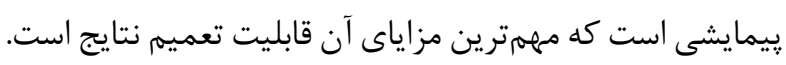

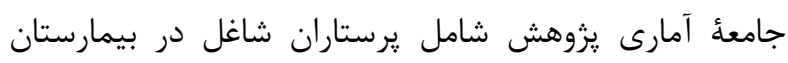

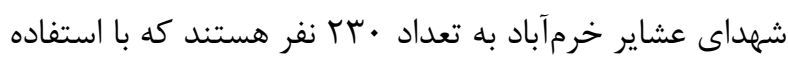

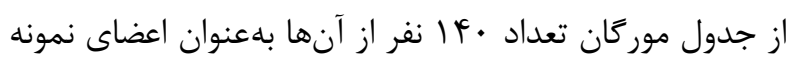

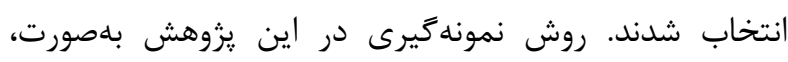

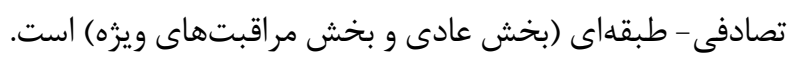

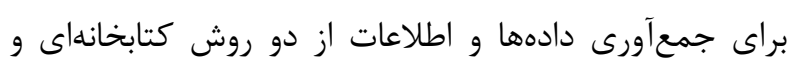

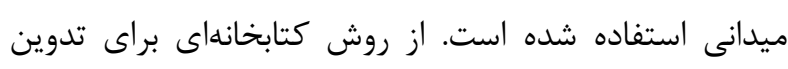

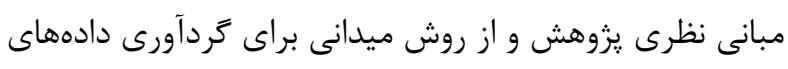

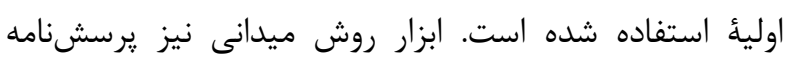

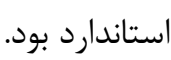

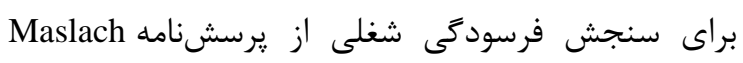

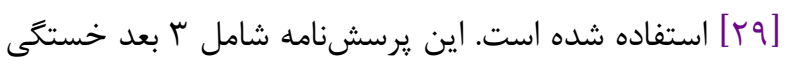

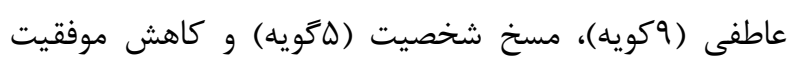

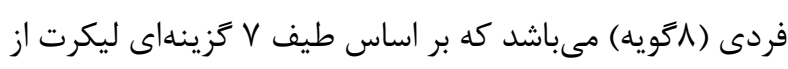

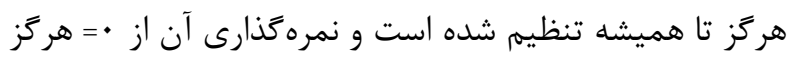

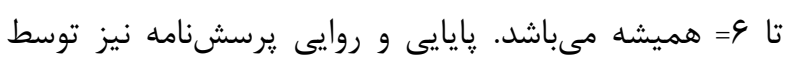
Barahuyi

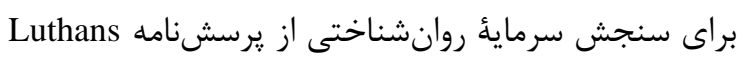

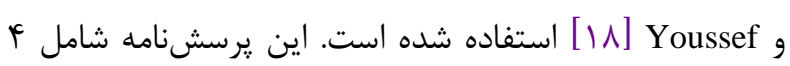


جدول ا. برازش مدلهاى اندازهيرى

\begin{tabular}{|c|c|c|c|}
\hline $\begin{array}{c}\text { ميانگين واريانس استخراجشده } \\
\text { (A VE) }\end{array}$ & هايايى تركيبى & آلفاى كرونباخ & متغير \\
\hline$\cdot 199 \mathrm{~V}$ & - $|\Lambda 9|$ & $\cdot / 1 \wedge 9$ & استرس ابتلا به كرونا \\
\hline$\cdot / V F$. & $\cdot|\Lambda| V$ & $\cdot / \wedge \Delta\rangle$ & فرسودگى شغلى \\
\hline . 1849 & $\cdot / V \varepsilon \wedge$ & ./V१६ & سرمايئ روانشناختى \\
\hline
\end{tabular}

بررسى مدل مفههومى تحقيق و آزمون فرضيه ها

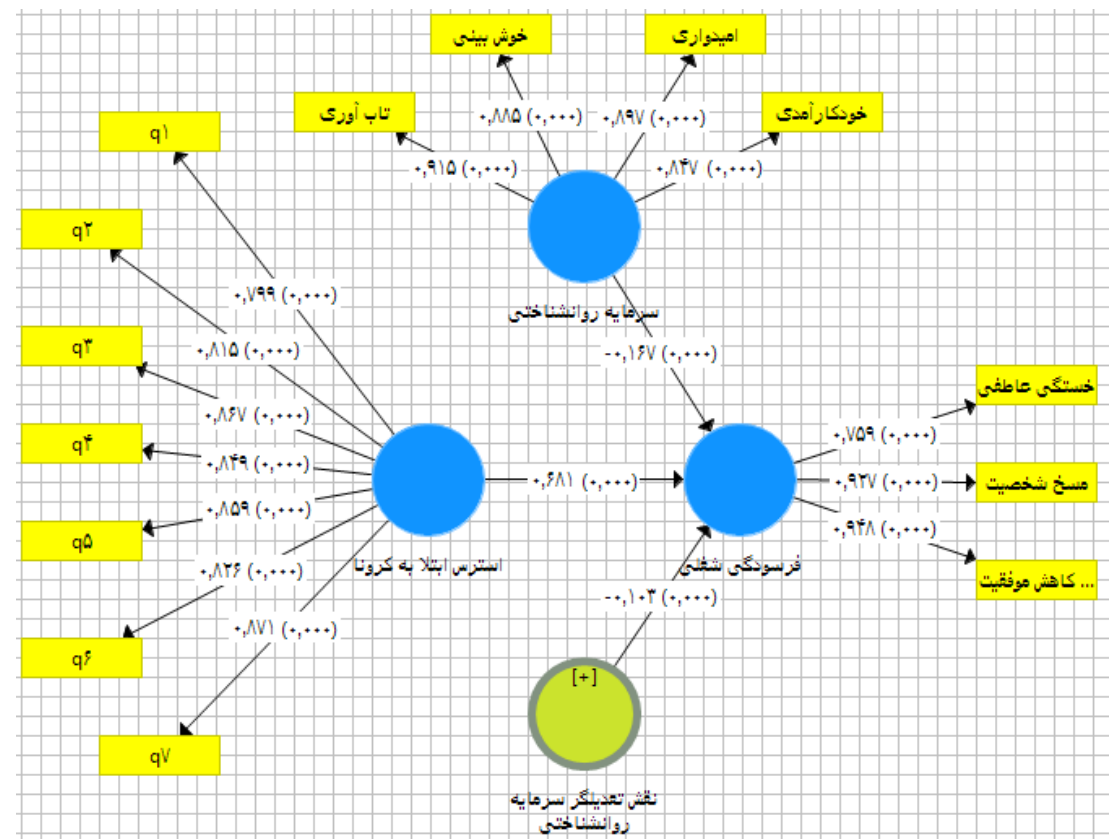

شكل r. مدل ترسيمشده همراه با مقادير P-value و ضرايب استاندارد

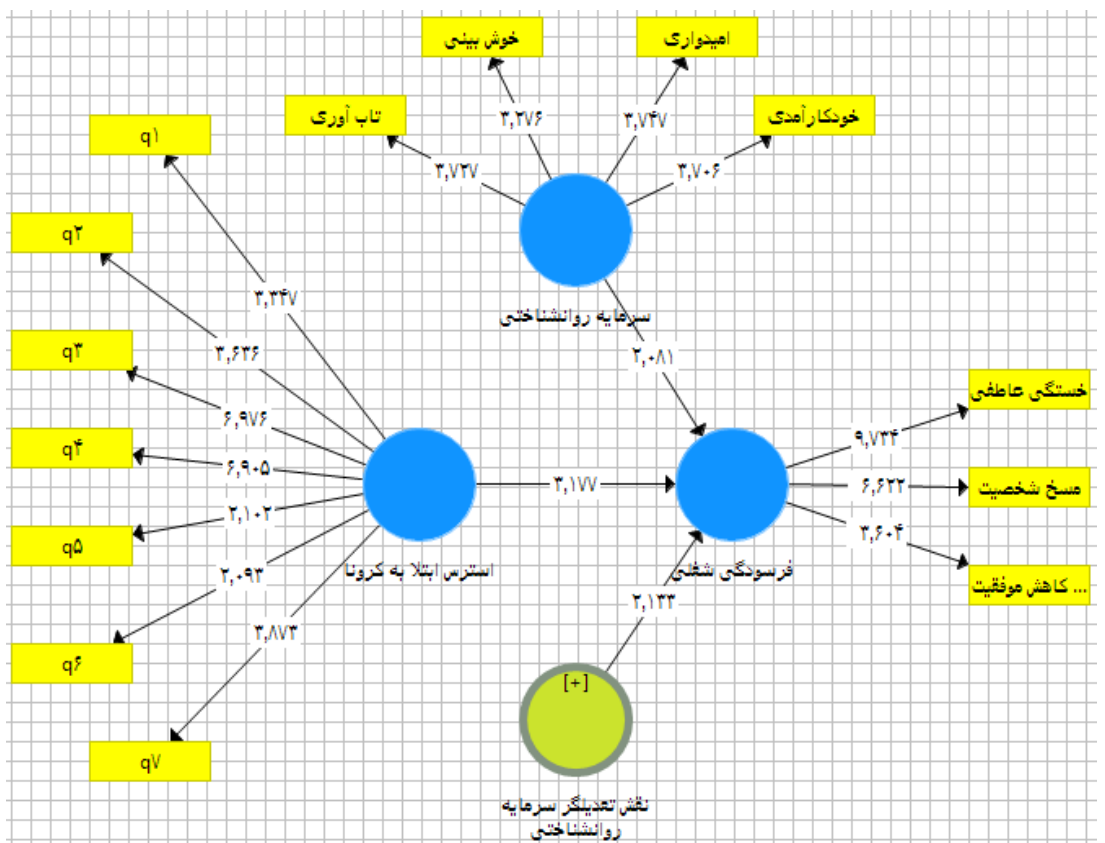

شكل r. مدل ترسيمشده همراه با مقادير آماره 
از جمله طولانى شدن زمان اين همهَيرى، ميزان بالاى

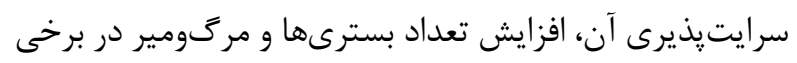

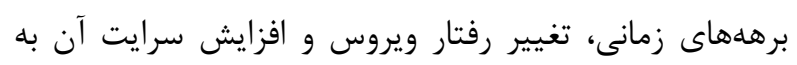

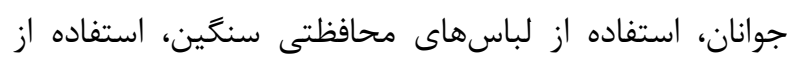

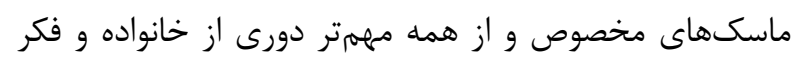

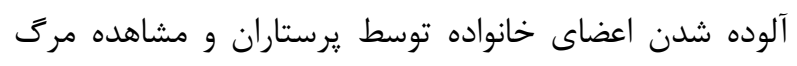

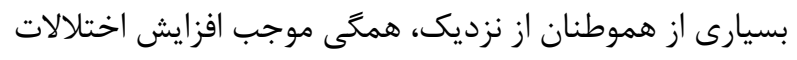

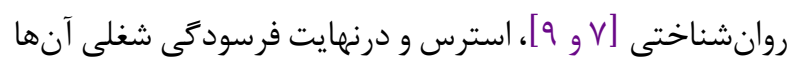

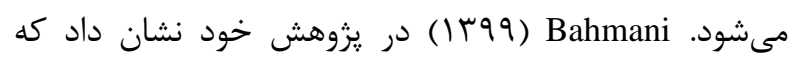

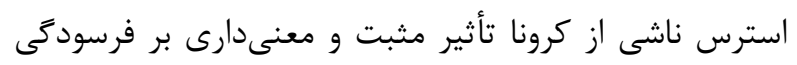

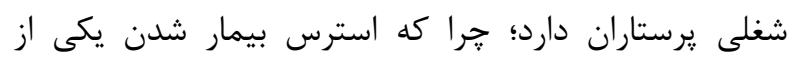
آزاردهندهترين وسواسهاست، استرس ابتلا به ويروس كراس كرونا

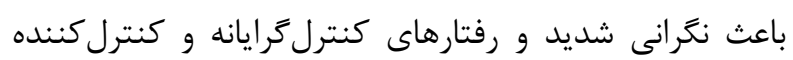

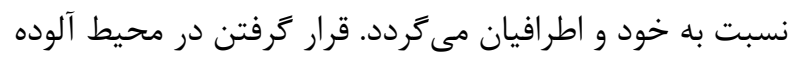

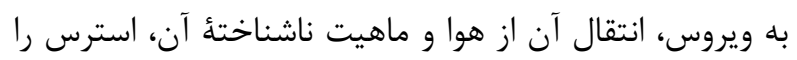

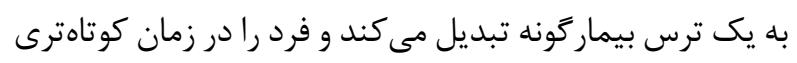

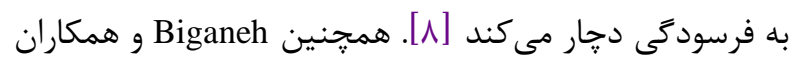

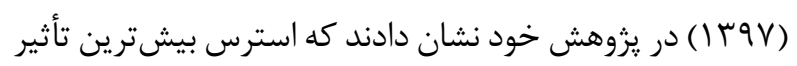

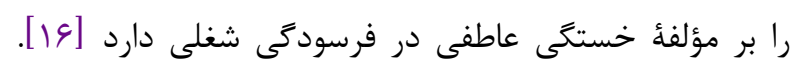
Sepahvand

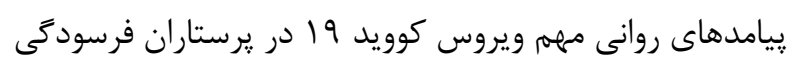

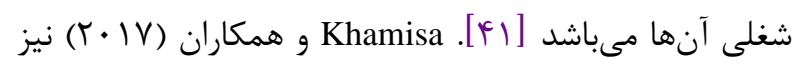
نشان دادند كه استرس كارى بر فرسودىى شغلى، رضايت شغلى آنى

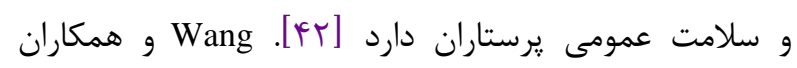

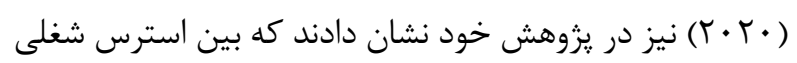

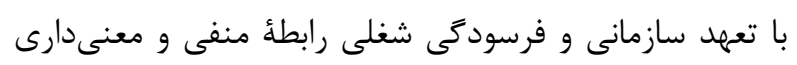
وجود دارد [سمان]. در رابطه با فرضئ دوم نيز مى دواردان كَتف كه ميزان

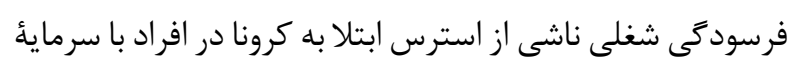

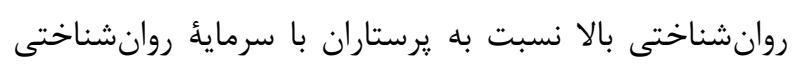

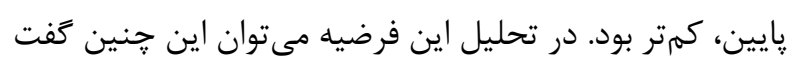

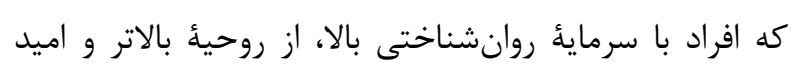

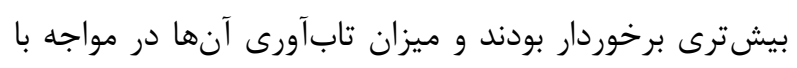

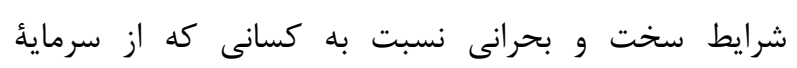

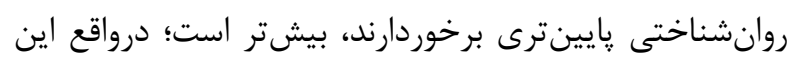

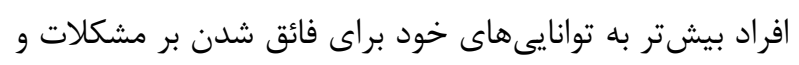

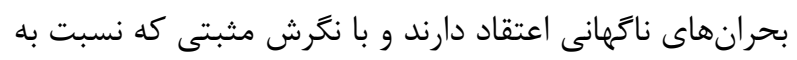

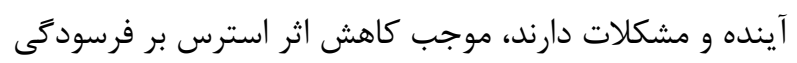

براى بررسى فرضيههاى يزوهش از مقادير T و ضرايب مسير

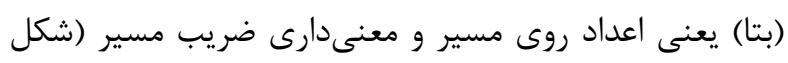

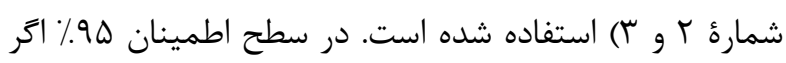

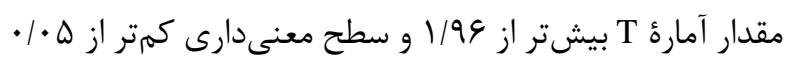
باشد فرضيه تأئيد مىشود. در فرضية اول كه به بررسى اثر استرس ابتلا به كرونا بر برد

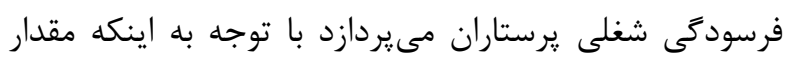

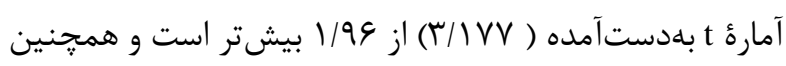

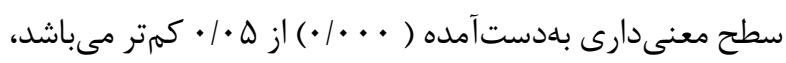
لذا اين فرضيه تأييد مى شود.

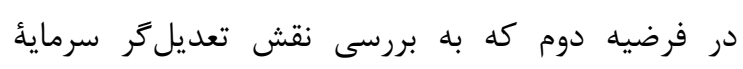

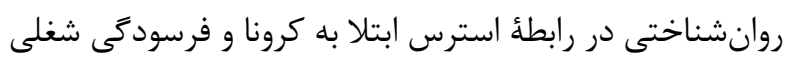

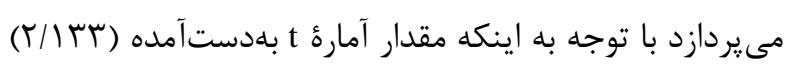

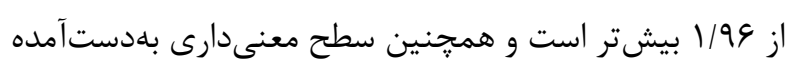

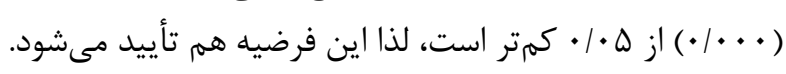

\section{بحث}

يروهش حاضر به بررسى رابطهٔ استرس ابتلا به كرونا بر

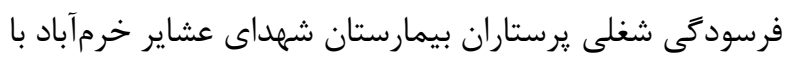
نقش تعديل

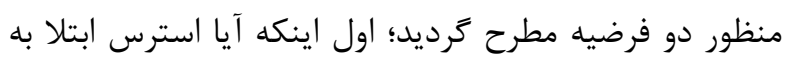

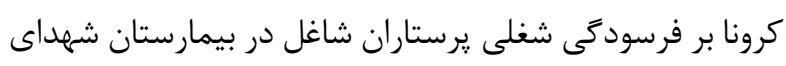

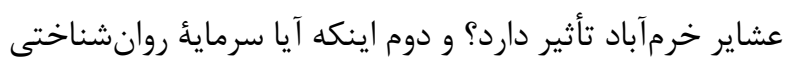

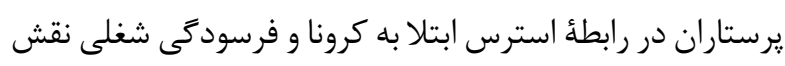
تعديلكر دارد يا خير؟

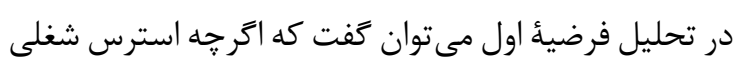

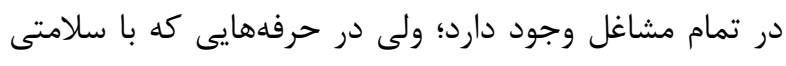

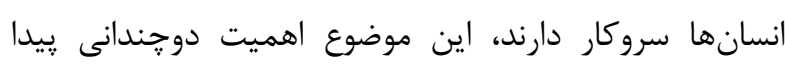

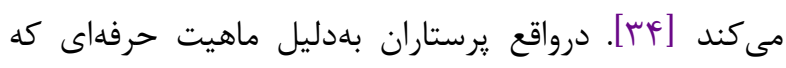

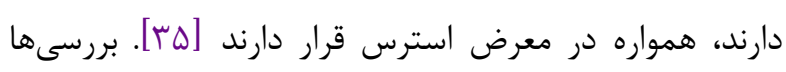

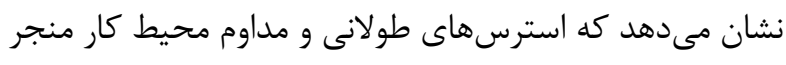

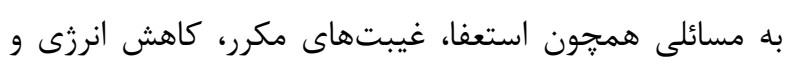

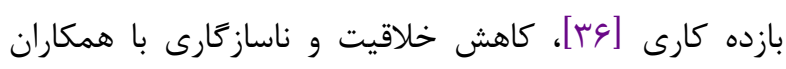

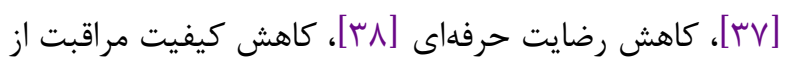

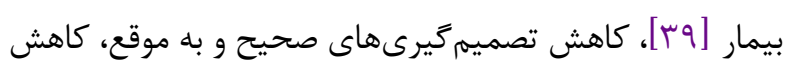

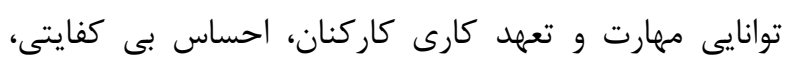

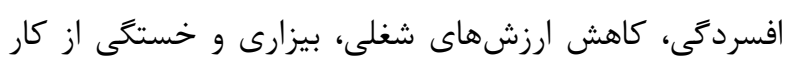

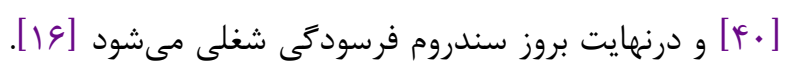

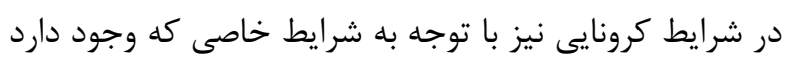


با توجه به اينكه استرس ابتلا به كرونا، هم تضعيف كننده سيستم

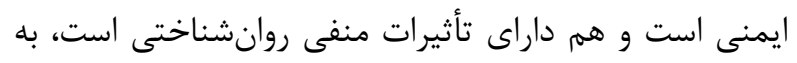

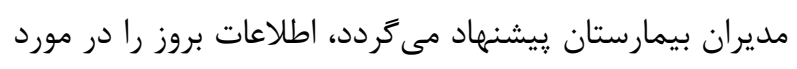

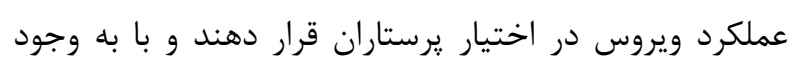

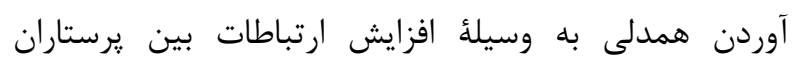
زمينههاى كاهش استرس را فراهم نمايند.

$$
\text { نتيجه كيرى زمينهاي كاهن }
$$

بلعنوان نتيجأ كلى يزوهش، مى توان كفت كه استرس ابتلا

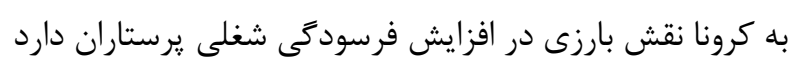

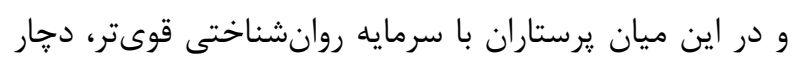

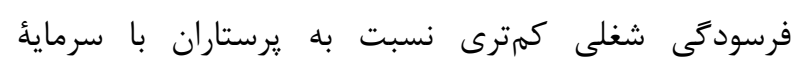

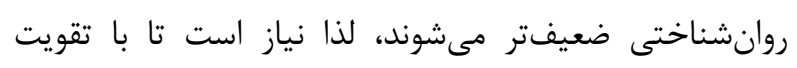

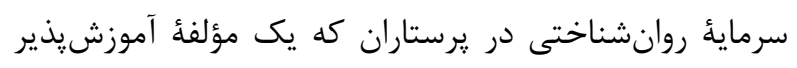

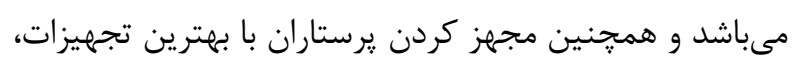

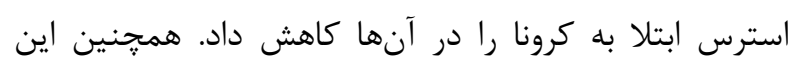

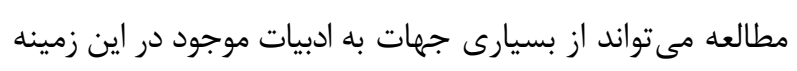

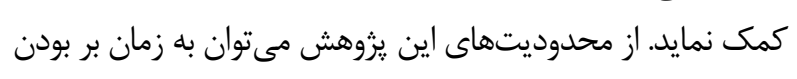

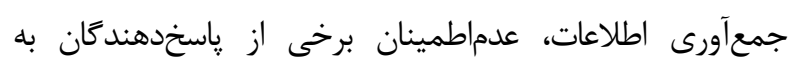

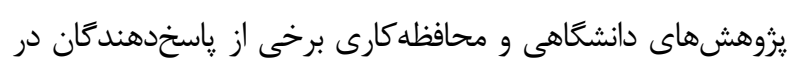

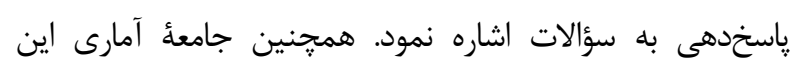
يزوهش، يرستاران شاغل در بيمارستان شهداى عشاير خرمآباد بودند،

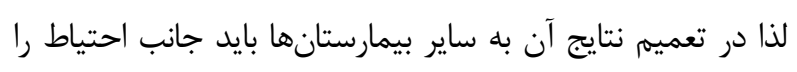

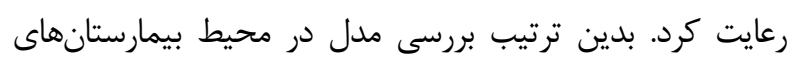
مختلف ممكن است نتايج متفاوتى داشته باشد.

\section{تقدير و تشكر}

ياد و خاطره تمام يرستاران عزيزى كه براثر ابتلا به بيمارى كرونا به شهداى سلامت بِيوستند را خرامى مى خداريم و از كليئ

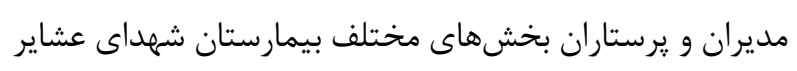

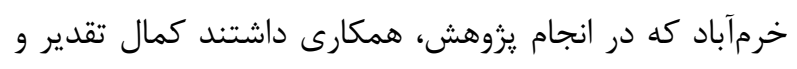

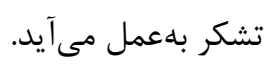

\section{تاييديه اخلاقى}

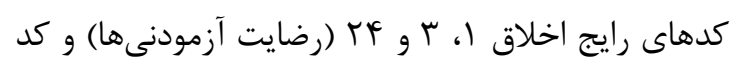

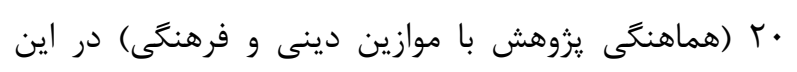
مطالعه رعايت شده است.
شغلى مىشوند. در واقع افراد با سرمائُ روانشناختى بالاتر اين

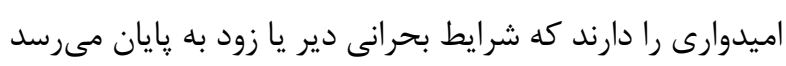

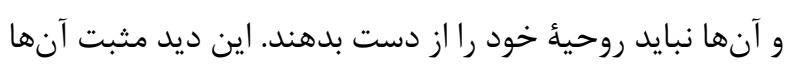

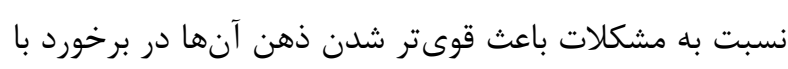

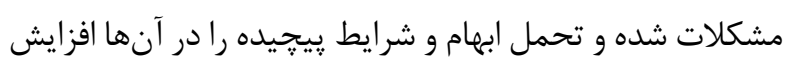

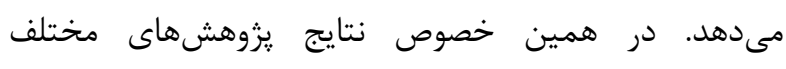

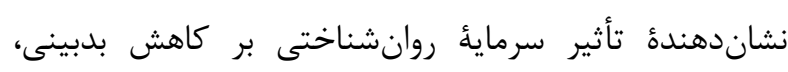

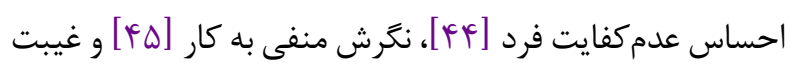

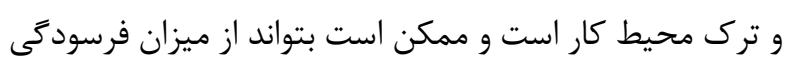

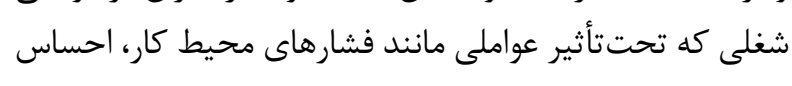

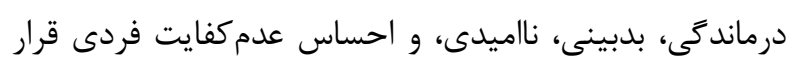

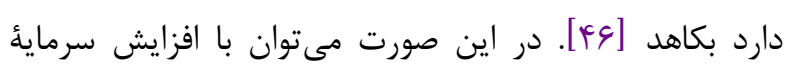

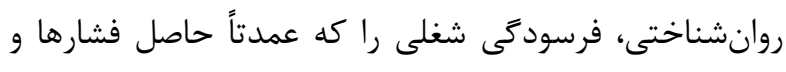

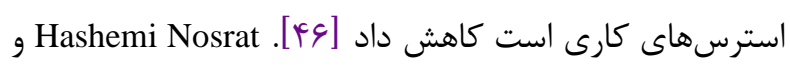

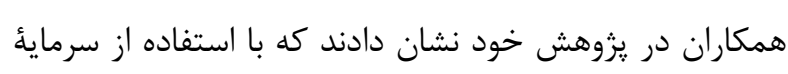

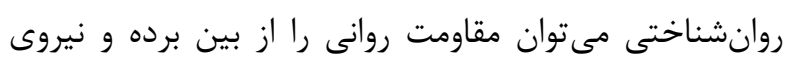

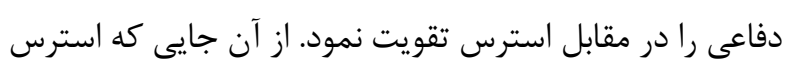

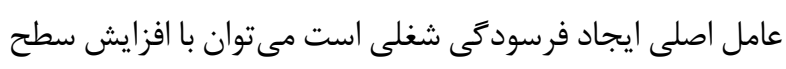

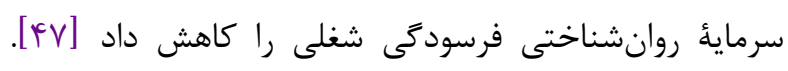
Silva

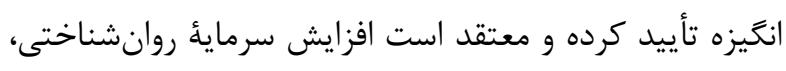

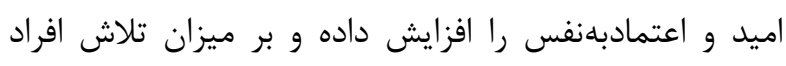

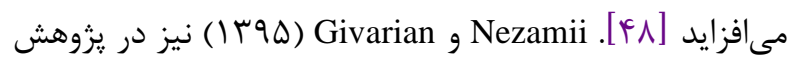

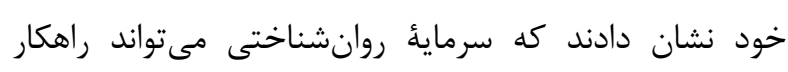

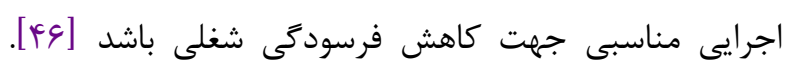

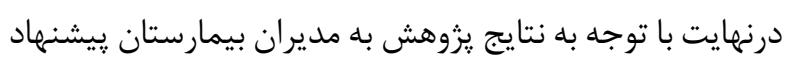

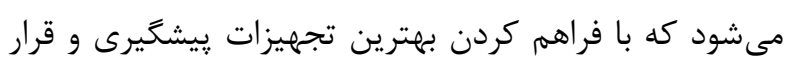

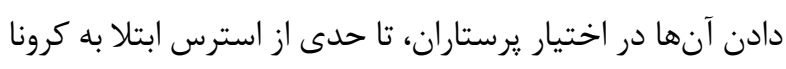

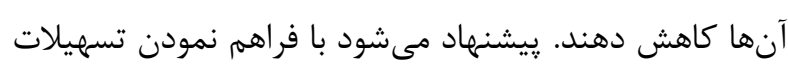

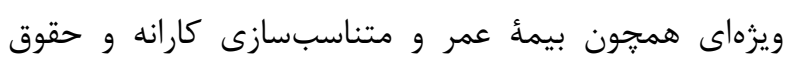

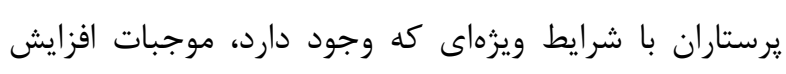

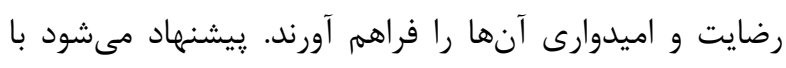

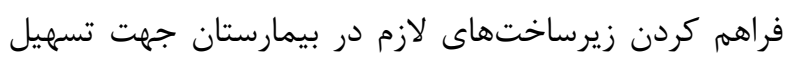

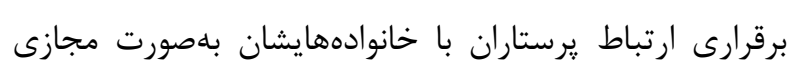

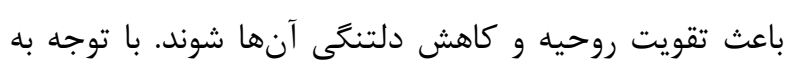

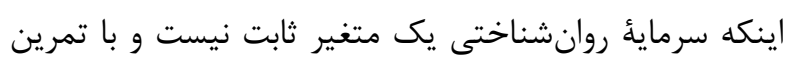

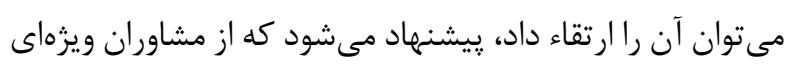

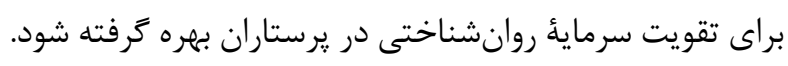


كلى، انتخاب رويكردها و نتيجه زيرى، مصطفى اميدنزاد؛ بررسى

$$
\text { نهايى و اصلاح مقاله. }
$$

$$
\text { منابع مالى }
$$

منابع مالى موردنياز براى انجام يزوهش حاضر را نويسندگان

$$
\text { تأمين كردهاند. }
$$

\section{References}

1. Li Q, Guan X, Wu P, Wang X, Zhou L, Tong Y, et al. Early transmission dynamics in Wuhan, China, of novel coronavirus-infected pneumonia. N Engl J Med. 2020.

2. Birghi Fard A, Kargar F. The Role of Lifestyle in Health and Prevention of Corona Disease. J Appl Stud Soc Sci. 2016;3(1):53.

3. Geller C, Varbanov M, Duval RE, Human coronaviruses: insights into environmental resistance and its influence on the development of new antiseptic strategies. Viruses. 2012;4(11). [DOI:10.3390/v4113044] [PMID] [PMCID]

4. The National Health Commission of China. Updates on the novel coronavirus outbreak up to March 2. 2020. Accessed March. 2020.www.behdasht.gov.ir

5. Anderson RM, Heesterbeek H, Klinkenberg D, Hollingsworth TD. How will country-based mitigation measures influence the course of the COVID-19 epidemic? The Lancet. 2020;102(28):931-4. [DOI:10.1016/S01406736(20)30567-5]

6. Sarboozi Hosein Abadi T, Askari M, Miri K, Namazi Nia M. Depression, stress and anxiety of nurses in COVID-19 pandemic in Nohe-Dey Hospital in Torbat-e-Heydariyeh city, Iran. J Mil Med. 2020;22(6):526-33

7. Waldron HA. Occupational health practice: ButterworthHeinemann. 2013.

8. Bahmani A. Investigating the effect of work shifts in coronary conditions on burnout of employees with the mediating role of coronary stress. Nurs Manag. 2020;9(4):20-26.

9. Nordang K, Hall-Lord M-L, Farup PG. Burnout in healthcare professionals during reorganizations and downsizing. A cohort study in nurses. BMC Nurs. 2010;9:8 [DOI:10.1186/1472-6955-9-8] [PMID] [PMCID]

10. Wu SY, Li HY, Wang XR, Yang SJ, Qiu H. A comparison of the effect of work stress on burnout and quality of life between female nurses and female doctors. Arch Environ Occup Health. 2011;66:193-200.

[DOI:10.1080/19338244.2010.539639] [PMID]

11. Piko BF. Burnout, role conflict, job satisfaction and psychosocial health among Hungarian health care staff: A questionnaire survey. Int J Nurs Stud. 2006;43:311-8. [DOI:10.1016/j.ijnurstu.2005.05.003] [PMID]

12. Abarghouei MR, Sorbi MH, Abarghouei M, Bidaki R, Yazdanpoor S. (2016). A study of job stress and burnout and related factors in the hospital personnel of Iran. Electron Physician. 2016;8(7):2625.

[DOI:10.19082/2625] [PMID] [PMCID]

\author{
تعارض منافع \\ بين نويسندكان هيجزَّنه تعارضى در منافع وجود ندارد. \\ سهم نويسند \\ دكتر محسن عارفنزاد؛ طراحى جارجوب كلى، تدوين محتوا و

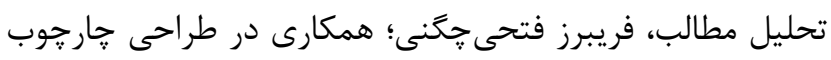

13. Kim J, Choi S. Factors influencing emergency nurses> burnout during an outbreak ofmiddle east respiratory syndrome coronavirus in korea. Asian Ners Res. 2016;11:1-5 [DOI:10.1016/j.anr.2017.01.001] [PMID]

14. Wu Y, Wang MD. A Comparison of Burnout Frequency Among Oncology Physicians and Nurses Working on the Frontline and usual wards during the COVID-19 epidemic in Wuhan. J Pain Symptom Manag. 2020;60(1):61 [DOI:10.1016/j.jpainsymman.2020.04.008] [PMID] [PMCID]

15. Mohammadi SZ, Haghighi SA. Relation between job stress and burnout among nursing staff. Avicenna Journal of Nursing and Midwifery Care. 2011;19(2):42-52.

16. Biganeh J, Abolghasemi J, Alimohammadi I, Ebrahimi H, Torabi Z, Ashtarinezhad, A. Survey of Occupational Stress Effects on Burnout among Nurses. J Knowledge Health. 2018;13(1):10-8.

17. Tabatabai S, Najafi Z, Hassanzadeh S. The relationship between sources of job stress and shift work of staff in a medical center in Tehran. In the third biennial conference on ergonomics in Iran. 2018.

18. Luthans F, Youssef CM. Emerging positive organizational behavior. J Manage. 2007;3(3):21-39. [DOI:10.1177/0149206307300814]

19. Luthans F, Youssef CM, Sweetman DS, Harms PD. Meeting the leadership challenge of employee well-being through relationship PsyCap and Health PsyCap. J Leadership Organ Stud. 2013;20(1):118-33. [DOI:10.1177/1548051812465893]

20. Luthans F, Norman S, Avolio B, Avey J. Supportive climate and organizational success: The mediating role of psychological capital. J Orga Behav. 2008;29(2):219-38. [DOI:10.1002/job.507]

21. Golmohammadian M, Sajjadi SN. The role of psychological capital and organizational citizenship behavior in predicting job burnout of exceptional elementary school teachers. Psychol Except Individ. 2017;6(24):109-33.

22. Youssef CM, Luthans F. An integrated model of psychological capital in the workplace. In P.A. Linley S. Harrington, \& N. Garcea (Eds.), Oxford handbook of positive psychology and work. New York: Oxford University Press. 2010:277-88.

23. Sun T, Zhao XW, Yang LB, Fan LH. The impact of psychological capital on job embeddedness and job performance among nurses: a structural equation approach. J Adv Nurs. 2012;68(1):69-79. [DOI:10.1111/j.13652648.2011.05715.x] [PMID] 
24. Herbert M. An exploration of the relationships between psychological capital (hope, optimism, self-efficacy, resilience), occupational stress, burnout and employee engagement. [MSc. of thesis] South Africa, University of Stellenbosch. 2011.

25. Liu L, Chang Y, Fu J, Wang J, Wang L. The mediating role of psychological capital on the association between occupational stress and depressive symptoms among Chinese physicians: a cross-sectional study. BMC Public Health. 2012;12(1):219-26. [DOI:10.1186/1471-2458-12219] [PMID] [PMCID]

26. Jiang X, Peng J, Zhang J, Xiao R, Song Y, Feng X, Zhang Y, Miao D. The impact of psychological capital on job burnout of Chinese nurses: the mediator role of organizational commitment. PloS one. 2013;8(12):e84193. [DOI:10.1371/journal. pone.0084193] [PMID] [PMCID]

27. Phili W, Karen-Leigh E. Caring as a resillient practice in Mental Health Nursing. Int $\mathbf{J}$ Ment Health Nurs. 2007;16(2):132-5.

28. Edward KL, Hercelinsky JG. Burnout in the caring nurse: Learning rsilient behaviors. Br J Nurs. 2007;(16):240-2. [DOI:10.1111/j.1447-0349.2007.00456.x] [PMID]

29. Maslach C, Schaufeli W, Leiter M. Job burnout. Annu Rev Psychol. 2001;52(1):397-22. [DOI:10.1146/annurev.psych.52.1.397] [PMID]

30. Barahuyi S, Nastiezaie N. The effect of work alienation on incidents organizational anti-citizenship behaviors through the mediating role of job burnout (Case study: principals of Zahedan high schools). Knowledge Res Appl Psychol. 2019;20(3):77-90.

31. Irajirad, A, Malekzadeh E. The effect of psychological capita Mediation by motivation on Students' Creativity. J Innov Creat Hum Sci. 2017;6(4):51-70.

32. Lin C, Ahorsu V, Saffari M, Griffiths MD., \& Pakpour AH. The Fear of COVID-19 Scale: Development and Initial Validation. Int J Ment Health Addict. 2020;27:1-9 [DOI:10.1007/s 11469-020-00270-8]

33. Rajabbaigy M, Bahmani A. The role of nurses' emotional burnout in coronavirus stress and work-related Accidents in Tehran Oil Industry Hospital. Hum Resourc Manag Oil Indus. 12(46):57-70

34. Hebrani P, Behdani F, Mobtaker M. Evaluation of stress factors in nurses different hospital wards. The Q J Fundam Mental Health. 2008;10(3):231-7.

35. Hosseini M. Fundamentals of nursing management. Tehran: Boshra. 2007.

36. Abdi H, Shahbazi L. Correlation between occupation stress in nurses intensive care unit with job burnout. J Shahid Sadoughi Univ Med Sci Health Services. 2001;9(3):58-63.

37. Meshkani ZS, Koohdani F. A study of anxiety among the nurses and its related factors. Daneshvar Med. 2004;11(52):47-52.
38. Sang Sook Han S, Soon Sohn I, Eun Kim N. New nurse turnover intention and influencing factors. Korean Acad Nurs. 2009;39(6):878-87. [DOI:10.4040/jkan.2009.39.6.878] [PMID]

39. Poghosyan L, Clarke SP, Finlayson M, Aiken LH. Nurse burnout and quality of care: crossnational investigation in six countries. Res Nurs Health. 2010;33(4):288-98. [DOI:10.1002/nur.20383] [PMID] [PMCID]

40. Enjezab B, Farnia F. Occupational stress \& psychologicalbehavioral responses. J Shahid Sadoughi Univ Med Sci Health Services. 2002;10(3):32-8.

41. Sepahvand R, Momeni Mofrad M, Taghipour S Identifying and prioritizing the psychological consequences of the Covid 19 virus in nurses. J Hosp. 2020;19(2):25-32.

42. Khamisa N, Peltzer K, Ilic D, Oldenburg B. (2017). Effect of personal and work stress on burnout, job satisfaction and general health of hospital nurses in South Africa. Health SA Gesondheid. 2017;22:252-8. [DOI:10.1016/j.hsag.2016.10.001]

43. Wang P, Chu P, Wang J, Pan R, Sun Y, Yan M,. et al. (2020). Association Between Job Stress and Organizational Commitment in Three Types of Chinese University Teachers: Mediating Effects of Job Burnout and Job Satisfaction. Front. Psychol. 2020;11:1-12. [DOI:10.3389/fpsyg .2020.576768] [PMID] [PMCID]

44. Jia-jun G. Research on a PCI model-based reform in college students' entrepreneurship education. Paper presented at: 2nd international conference on Artificial Intelligence, Management Science and Electronic Commerce (AIMSEC) .2011. [DOI:10.1109/AIMSEC.2011.6010770]

45. Luthans F, Avey JB, Avolio BJ, Peterson SJ. The development and resulting performance impact of positive psychological capital. Hum Resour eDve Q. 2010;21 (1):41-67. [DOI:10.1002/hrdq .20034]

46. Nezamii A, Givarian H. An investigation of the effect of psychological capital on the burnout of nurses in a military hospital in Tehran. Ebnesina- IRIAF Health Administration. 2016;18(1):44-51.

47. Hashemi Nosrat Abad T, Babapur Kheyroddin J, Bahadori Khosroshahi J. Role of psychological capital in psychological well-being by considering the moderating effects of social capital. Soc Psychol Res. 2012;1(4):123144.

48. Silva D, Roche M. Contribution of psychological capital to entrepreneurs success during recessionary times. New perspectives in business research and education: New Zealand Applied Business Education Conference (NZABE). 2010; Napier, New Zealand. 